\title{
Prevalencia de mutación germinal de BRCA en mujeres jóvenes con cáncer de mama: experiencia de un Centro Privado de Tercer Nivel
}

\author{
Prevalence of BRCA germinal mutation among young women \\ with breast cancer:" experience in a Third Level Private Center
}

\author{
Daniela Vázquez Juárez, ${ }^{*}$ Juan Alberto Serrano Olvera, ${ }^{\ddagger}$ \\ Alejandro Noguez Ramos, ${ }^{\S}$ Gabriela Regalado Porras, ${ }^{\ddagger}$ Jesús M Lázaro León,,$\mp$ \\ Guillermo Olivares Beltrán, ${ }^{\ddagger}$ Raquel Gerson Cwilich ${ }^{\ddagger}, \mathbb{T}$
}

Citar como: Vázquez JD, Serrano OJA, Noguez RA, Regalado PG, Lázaro LJM, Olivares BG et al. Prevalencia de mutación germinal de BRCA en mujeres jóvenes con cáncer de mama: experiencia de un Centro Privado de Tercer Nivel. An Med ABC. 2021 ; 66 (4): 249-257. https://dx.doi.org/10.35366/102924

\section{RESUMEN}

Introducción: $\mathrm{El}$ cáncer de mama en mujeres jóvenes tiene un comportamiento y biología asociados con mayor riesgo de recurrencia y muerte. El diagnóstico de cáncer de mama en mujeres jóvenes guarda fuerte asociación con la presencia de mutaciones genéticas, principalmente en el gen BRCA. Sin embargo, no se ha observado una asociación entre la presencia de mutaciones genéticas heredadas y el pronóstico. Objetivo: Describir la prevalencia y analizar las características clínicopatológicas de mujeres, < 50 años con cáncer de mama, con y sin mutación germinal de BRCA. Material y métodos: Estudio descriptivo, observacional, transversal de mujeres < 50 años con cáncer de mama con y sin mutación germinal de BRCA que recibieron tratamiento en un Centro Médico Privado de Tercer Nivel. Resultados: Se identificaron 53 mujeres con cáncer de mama $<50$ años y resultado de panel genético. La mediana de edad fue 40 años $(27-50), 55 \%$ fue $<40$ años y 30/53 (56.6\%) reportaron mutaciones en el panel genético. Mutación de BRCA1 y/o BRCA2 en nueve/30 (30\%), todas variantes patogénicas. Mutaciones diferentes a BRCA en 21/30

\section{ABSTRACT}

Introduction: Breast cancer in young women has a behavior and biology associated with an increased risk of recurrence and death. The diagnosis of breast cancer in young women is strongly associated with the presence of genetic mutations, mainly in the BRCA gene. However, an association between the presence of inherited genetic mutations and prognosis has not been observed. Objective: To describe the prevalence and analyze the clinical-pathological characteristics of women, $<$ 50 years with breast cancer, with and without BRCA germline gene mutation. Material and methods: A descriptive, observational, cross-sectional study of women $<50$ years with $B C$ with and without BRCA germline mutation who received treatment at a Private Third Level Medical Center. Results: 53 women with $B C<50$ years and a genetic panel result were identified. Median age was 40 years $(27-50), 55 \%<40$ years old. 30/53 women (56.6\%), presented some mutation in the genetic panel. BRCA1 and/or BRCA2 9/30 (30\%) mutation, all of them were pathogenic variants. Mutations other than BRCA in $21 / 30(70 \%)$, of these $7 / 21(33.3 \%)$ pathogenic mutations in the
* Oncología Médica, Centro de Cáncer de Mama, Hospital Zambrano-Hellion TecSalud; Monterrey, Nuevo León.

‡ Oncología Médica, Centro de Cáncer Hospital ABC. Ciudad de México.

$\S$ Oncología Médica, Clínica de tumores gastrointestinales, Instituto Nacional de Ciencias Médicas «Salvador Zubirán». Ciudad de México.

१ Directora Centro de Cáncer, Centro Médico ABC, Ciudad de México.
Recibido: 03/06/2021. Aceptado: 16/11/2021.

Correspondencia:

Juan Alberto Serrano Olvera

E-mail: serranoolvera@gmail.com 
(70\%), de éstas siete/21 (33.3\%) fueron mutaciones patogénicas en los genes ATM (2), MUTYH (2), TP53 (2) y PALB2 (1). En $14 / 21$ (66.7\%) se identificaron variantes de significado incierto. No observamos asociación entre las características clínico-patológicas y el estado mutacional de BRCA u otras mutaciones genéticas, excepto el alto grado de diferenciación $(p=0.04)$, siendo más frecuente en el grupo mutado. No hubo diferencias en el tiempo libre de enfermedad entre las pacientes BRCA mutados y no mutados $(\mathrm{p}=0.12)$. Conclusiones: La prevalencia de la mutación germinal de BRCA en mujeres con cáncer de mama $<50$ años en nuestra población fue de $30 \%$. Las características clínicas, biológicas y el tiempo libre de enfermedad no difirieron entre el grupo con y sin mutación germinal de BRCA ni de otros genes.

Palabras clave: Cáncer de mama, mujeres jóvenes, mutación germinal BRCA.

Nivel de evidencia: II

\section{INTRODUCCIÓN}

El cáncer de mama $(\mathrm{CM})$ es la neoplasia que con más frecuencia se diagnostica y la principal causa de muerte por cáncer en mujeres en México y en el mundo. En 2018 se diagnosticaron aproximadamente 2.1 millones de casos nuevos de CM y 627,000 muertes por esta enfermedad, lo que representa $6 \%$ de muertes por cáncer en mujeres de manera global. ${ }^{1}$

Actualmente, se calcula que $7 \%$ de las mujeres con $\mathrm{CM}$ se diagnostica antes de los 40 años y diversos estudios registran una biología tumoral y comportamiento más agresivo asociado a un pronóstico menos favorable en ese subgrupo de pacientes. ${ }^{2}$ En América Latina, la edad promedio al momento del diagnóstico es por lo general 10 años menor que la descrita en los países desarrollados y esto se ve reflejado en mayor proporción de pacientes $<40 \mathrm{y}<50$ años con $\mathrm{CM}$, con tasas que alcanzan 11 y $20 \%$, respectivamente. ${ }^{2}$ Otro estudio reportó que en Latinoamérica y países del Caribe la proporción de mujeres con $\mathrm{CM}<40$ años varía de 8.2 a 14\%. ${ }^{3}$ En México, durante el año 2001 un estudio reveló una prevalencia de $16.4 \%$ en mujeres $<40$ años. ${ }^{4}$

La definición de «mujer joven» en la oncología es muy variable, tanto las guías de EUSOMA (Sociedad Europea de Especialistas en CM) como BCY1 (cáncer de mama en mujeres jóvenes) definen a las «mujeres jóvenes» a las menores de 40 años. Para fines de tratamiento, St. Gallen establece el corte en mujeres menores de 35 años. ${ }^{5}$ Múltiples estudios han definido diferentes niveles de corte que van desde mujeres menores de 30 años hasta incluso menores 50 años como «jóvenes». 6,7
ATM (2), MUTYH (2), TP53 (2) and PALB2 (1) genes. In 14/21 $(66.7 \%)$ variants of uncertain significance were identified. We did not observe an association between the clinicopathological characteristics and the BRCA mutational status or other genetic mutations, except for the high degree of differentiation ( $p=$ 0.04 ), being more frequent in the mutated group. There were no differences in disease-free time between BRCA mutated and non-mutated patients $(p=0.12)$. Conclusions: The prevalence of the BRCA germline mutation in women with breast cancer $<$ 50 years in our population was $30 \%$. The clinical and biological characteristics and the disease-free time were not different among the group with and without BRCA germline mutation, or other genes.

Keywords: Breast cancer, young women, BRCA germline mutation.

Level of evidence: II

Las investigaciones sugieren que «las pacientes jóvenes» presentan neoplasias poco diferenciadas y un mayor porcentaje de infiltración vascular, comparadas con las pacientes de mayor edad; además en estas pacientes jóvenes se encontró mayor cantidad de casos con metástasis a ganglios axilares. ${ }^{8}$

Los factores pronósticos desfavorables se observan con mayor frecuencia en mujeres jóvenes en comparación con sus pares mayores. Al momento del diagnóstico, entre las mujeres jóvenes, las lesiones son más grandes, indiferenciadas y con menos proporción expresan receptores hormonales, pero relativamente mayores casos de sobreexpresión de HER2 e invasión vascular. ${ }^{9}$

Además, el CM en este grupo de edad provoca la investigación genética de síndromes del cáncer hereditario. BRCA1 y BRCA2 son genes supresores de tumores, los cuales se ubican en los cromosomas 17q21 y 13q12, respectivamente. Las proteínas BRCA funcionales están involucradas en el mantenimiento de la estabilidad del genoma por medio de la reparación de rupturas de doble cadena del ácido desoxirribonucleico (ADN), a través el sistema de recombinación homóloga. ${ }^{10}$

Actualmente, se estima que entre 3 y $10 \%$ de los casos de CM tienen un componente hereditario y de ellos $90 \%$ se asocia con mutaciones genéticas de alta penetrancia como la de los genes de BRCA1 y BRCA2. El resto se explica por mutaciones de genes de penetrancia intermedia como ATM, BARD1, PALB2 y CHECK2. ${ }^{11,12}$ En México son escasos los datos disponibles de CM con BRCA mutado. Distintas cohortes establecen una proporción entre 15-23\%; y la mutación fundadora mexicana en BRCA1 (deleción de los exones 9 a 12) representa hasta $30 \%$ del total de las mutaciones identificadas. ${ }^{13,14}$ 
A pesar del conocimiento que se tiene sobre los genes de BRCA, aún no está claro si una mutación de la línea germinal BRCA1 o BRCA2 tiene implicaciones pronósticas independientes. Los estudios y metaanálisis publicados han mostrado resultados discordantes con respecto al pronóstico de las mujeres con mutación en los genes de BRCA en comparación con pacientes con CM esporádico. Creemos que identificar el grupo de pacientes con mayor riesgo de CM secundario a una mutación germinal en los genes de BRCA puede permitir el desarrollo de estrategias para la prevención, diagnóstico y tratamiento para las pacientes afectadas y los miembros de la familia.

Objetivo: describir la prevalencia y analizar las características clínico-patológicas de mujeres $<50$ años con cáncer de mama con y sin mutación germinal de BRCA.

\section{MATERIAL Y MÉTODOS}

Se realizó un estudio descriptivo, observacional, transversal, único céntrico.

Los criterios de inclusión fueron mujeres de 18 a 50 años, con diagnóstico de CM invasor histológicamente confirmado que cuenten con resultado de prueba genética para mutación germinal de BRCA 1/2 mediante cualquier plataforma de secuenciación, atendidas y tratadas en el Centro Médico.

Se recolectaron las variables sociodemográficas de cada caso y las variables clínico-patológicas de cada neoplasia; también se registró el tiempo a la recurrencia de la enfermedad, el cual se definió como el tiempo del diagnóstico a la recurrencia documentada. Se utilizó estadística descriptiva con medidas de tendencia central. Mediante la fórmula para estimar una proporción, se calculó un tamaño de muestra de 51 pacientes para un poder estadístico de $80 \%$ con un error alfa de 0.05 , considerando una prevalencia de mutación de BRCA de 10\%.

Para contrastar las diferencias entre las variables cuantitativas se utilizó t de Student o bien U de Mann-Whitney para la distribución normal o libre distribución, respectivamente; para variables cuantitativas se empleó $\chi^{2}$ y frente a distribuciones se utilizaron pruebas no paramétricas. Los datos fueron analizados mediante el programa estadístico SPSS versión 22.

\section{RESULTADOS}

Durante el periodo comprendido del 01 de enero de 2015 al 01 de junio de 2020 se identificaron 807 mu- jeres con diagnóstico de cáncer de mama que recibieron tratamiento sistémico bajo indicación adyuvante, neoadyuvante o paliativa. De ellas, 360 (44.6\%) tenían 50 años o menos. Después del análisis de expedientes se detectó un resultado de panel genético en 91 de 360 solicitudes, en esos reportes al menos se incluyó el estado de los genes BRCA 1/2. Sin embargo, el estudio se documentó sólo en 53 casos, los cuales son el total de la muestra que integra este trabajo. De las 53 mujeres estudiadas, nueve (16.9\%) presentaron mutación de los genes de BRCA1 y/o BRCA2.

En las 53 mujeres incluidas en el análisis, la mediana de edad fue 40 años (27-50); 38 (27-48) años en las mujeres con mutación en BRCA1/2 y 40 (2850) años en aquéllas sin mutación. En el grupo con mutación de BRCA1/2 cinco mujeres (55.5\%) tenían 40 años o menos, la distribución de casos en el grupo sin mutación fue $23(52 \%)$ y $21(48 \%)$ para mujeres menores y mayores de 40 años, respectivamente. Se registraron antecedentes heredofamiliares de cáncer en $42 / 53$ pacientes $(79.2 \%)$, la mediana de familiares afectados fue dos (de uno a tres). Tres pacientes (7.1\%) informaron relación consanguínea en primer grado; en otros $11(26.2 \%)$ familiares en primer y/o segundo grado y 28 (66.7\%) familiares en primero y/o segundo y/o tercer grado.

Se identificó historia familiar de cáncer en sitios anatómicos asociados a la mutación germinal de BRCA 1/2 (mama, estómago, colon, páncreas, próstata) en tres pacientes $(7.1 \%)$ con relación consanguínea en primer grado; en ocho (19.1\%) familiares en primer y/o segundo grado; en $16(38.1 \%)$ familiares en primero y/o segundo y/o tercer grado; y en $15(35.7 \%)$ en sitios anatómicos sin asociación a la mutación en los genes de BRCA. Las características clínico-patológicas más relevantes se enumeran en la Tabla 1.

El inmunofenotipo, de acuerdo con la evaluación por inmunohistoquímica de los receptores hormonales, Ki-67 y estado de HER2/neu, fue luminal A en $13(24.5 \%)$ mujeres, luminal B en 18 (34\%), luminal B con sobreexpresión de HER2/neu en siete (13.2\%), HER2/neu puro en tres (5.7\%) y en $12(22.6 \%)$ casos fue triple negativo.

Receptores de estrógeno positivos se identificaron en $38(71.7 \%)$, con un porcentaje medio de expresión de $47 \%$ (1-100\%). Receptores de progesterona en 39 (73.6\%) y el porcentaje medio de expresión fue de $33 \%$ (1-100\%). La media de Ki67 en todas las mujeres estudiadas fue de 38\% (1-90\%). HER2/neu por inmunohistoquímica fue positivo en siete $(13.2 \%)$ mujeres, indeterminado $(2+)$ en cinco $(9.4 \%)$ y negativo en 41 
Tabla 1: Características clínico-patológicas de la población. $N=53$

\begin{tabular}{|c|c|c|c|}
\hline & $\begin{array}{l}\text { Todas } \\
\mathrm{n}(\%)\end{array}$ & $\begin{array}{c}\text { Con mutación BRCA1/2 }(\mathrm{N}=9) \\
\mathrm{n}(\%)\end{array}$ & $\begin{array}{c}\text { Sin mutación }(\mathrm{N}=44) \\
\mathrm{n}(\%)\end{array}$ \\
\hline Edad (años)* & $40(27-50)$ & $38(27-48)$ & $40(28-50)$ \\
\hline$<30$ & $4(7.5)$ & $2(22.2)$ & $2(4.6)$ \\
\hline $30-40$ & $24(45.3)$ & $3(33.3)$ & $21(47.7)$ \\
\hline $41-50$ & $25(47.2)$ & $4(44.5)$ & $21(47.7)$ \\
\hline \multicolumn{4}{|l|}{ Menopausia } \\
\hline Sí & 7 (12.2) & $0(0.0)$ & $7(15.9)$ \\
\hline No & $46(86.8)$ & $9(100.0)$ & $37(84.1)$ \\
\hline \multicolumn{4}{|c|}{ Índice de masa corporal $\left(\mathrm{kg} / \mathrm{m}^{2}\right)$} \\
\hline$<18.5$ & $4(7.5)$ & $1(11.1)$ & $3(6.8)$ \\
\hline $18 \leq 25$ & $30(56.6)$ & $5(55.6)$ & $25(56.8)$ \\
\hline $25 \leq 30$ & $16(30.2)$ & $3(33.3)$ & $13(29.6)$ \\
\hline$>30$ & $3(5.7)$ & $0(0.0)$ & $3(6.8)$ \\
\hline \multicolumn{4}{|l|}{ Tamaño tumoral } \\
\hline$T x$ & $1(1.9)$ & $1(11.1)$ & $0(0.0)$ \\
\hline $\mathrm{T} 1$ & $21(39.6)$ & $3(33.3)$ & $18(40.9)$ \\
\hline T2 & $25(47.2)$ & $4(44.5)$ & $21(47.7)$ \\
\hline T3 & $2(3.8)$ & $0(0.0)$ & $2(4.6)$ \\
\hline T4 & $4(7.5)$ & $1(11.1)$ & $3(6.8)$ \\
\hline \multicolumn{4}{|l|}{ Ganglios axilares } \\
\hline No & $29(54.8)$ & $3(33.3)$ & $26(59.1)$ \\
\hline N1 & $14(26.4)$ & $2(22.3)$ & $12(27.3)$ \\
\hline N2 & $5(9.4)$ & $3(33.3)$ & $2(4.5)$ \\
\hline N3 & $5(9.4)$ & $1(11.1)$ & $4(9.1)$ \\
\hline \multicolumn{4}{|l|}{ Metástasis } \\
\hline MO & $48(90.6)$ & $9(100.0)$ & $39(88.6)$ \\
\hline M1 & $5(9.4)$ & $0(0.0)$ & $5(11.4)$ \\
\hline \multicolumn{4}{|l|}{ Lateralidad } \\
\hline Derecha & $22(41.5)$ & $2(22.2)$ & $20(45.4)$ \\
\hline Izquierda & $30(56.6)$ & 7 (77.3) & $23(52.3)$ \\
\hline Bilateral & $1(1.9)$ & $0(0.0)$ & $1(2.3)$ \\
\hline \multicolumn{4}{|l|}{ Tipo histológico } \\
\hline Ductal & $50(94.3)$ & $9(100.0)$ & 41 (93.2) \\
\hline Lobulillar & $1(1.9)$ & $0(0.0)$ & $1(2.3)$ \\
\hline Mixto & $1(1.9)$ & $0(0.0)$ & $1(2.3)$ \\
\hline Medular & $1(1.9)$ & $0(0.0)$ & $1(2.3)$ \\
\hline \multicolumn{4}{|l|}{ Grado histológico } \\
\hline 1 & $2(3.8)$ & $0(0.0)$ & $2(4.5)$ \\
\hline 2 & $27(50.9)$ & $3(33.3)$ & $24(54.6)$ \\
\hline 3 & $21(39.6)$ & $5(55.6)$ & $16(36.4)$ \\
\hline No reportado & $3(5.7)$ & $1(11.1)$ & $2(4.5)$ \\
\hline \multicolumn{4}{|c|}{ Invasión linfovascular } \\
\hline Presente & $24(45.3)$ & $4(44.4)$ & $20(44.4)$ \\
\hline Ausente & $21(39.6)$ & $5(55.6)$ & $16(36.4)$ \\
\hline No reportado & $8(15.1)$ & $0(0.0)$ & $8(18.2)$ \\
\hline \multicolumn{4}{|l|}{ Invasión perineural } \\
\hline Presente & $5(9.4)$ & 2 (22.2) & $3(6.8)$ \\
\hline Ausente & $40(75.5)$ & $7(77.3)$ & $33(75.0)$ \\
\hline No reportado & $8(15.1)$ & $0(0.0)$ & $8(18.2)$ \\
\hline
\end{tabular}

${ }^{*}$ Mediana. 
Tabla 2: Características moleculares. $\mathrm{N}=53$.

\begin{tabular}{|c|c|c|c|}
\hline & $\begin{array}{l}\text { Todas } \\
\mathrm{n}(\%)\end{array}$ & $\begin{array}{l}\text { Con mutación BRCA1/2 }(\mathrm{N}=9) \\
\mathrm{n}(\%)\end{array}$ & $\begin{array}{c}\text { Sin mutación }(\mathrm{N}=44) \\
\mathrm{n}(\%)\end{array}$ \\
\hline \multicolumn{4}{|l|}{ Inmunofenotipo } \\
\hline Luminal B (RH+/HER2-) & $18(34.0)$ & $3(33.3)$ & $15(34.1)$ \\
\hline Luminal B (RH+/HER2+) & 7 (13.2) & $1(11.1)$ & $6(13.7)$ \\
\hline \multicolumn{4}{|l|}{ Receptores de estrógeno } \\
\hline Positivo & $38(71.7)$ & $7(77.3)$ & $31(70.5)$ \\
\hline Negativo & $15(28.3)$ & $2(22.2)$ & $13(29.5)$ \\
\hline \multicolumn{4}{|l|}{ Receptores de progesterona } \\
\hline Positivo & $39(73.6)$ & $8(88.9)$ & $31(70.5)$ \\
\hline Negativo & $14(26.4)$ & $1(11.1)$ & $13(29.5)$ \\
\hline$<20$ & $16(30.2)$ & $3(33.3)$ & $10(22.7)$ \\
\hline$\geq 20$ & $34(64.1)$ & $6(66.7)$ & $31(70.5)$ \\
\hline No reportado & $3(5.7)$ & $0(0.0)^{\prime}$ & $3(6.8)$ \\
\hline
\end{tabular}

(77.4\%). A todas las mujeres con resultado indeterminado por inmunohistoquímica se determinó el estado de HER2/neu por técnicas de inmunofluorescencia, con resultado positivo en tres/cinco, indeterminado una/cinco y negativo en una/cinco (Tabla 2).

$\mathrm{Al}$ contrastar las características clínico-patológicas entre las mujeres con y sin mutación en los genes de BRCA1/2, el grado histológico 3 fue la única variable que mostró tener una diferencia estadísticamente significativa, 55.6 versus $36.4 \%, \mathrm{p}=0.04$ al ser más frecuente en el grupo con mutación. No se encontró diferencia en relación con la edad, antecedentes heredofamiliares de cáncer, características clínicas del cáncer y patológicas e inmunohistoquímicas al comparar aquéllas con y sin mutación de los genes estudiados.

El ADN genómico se extrajo de muestras de sangre en $34(64 \%)$ mujeres y a partir de saliva en 19 (36\%). Las plataformas utilizadas fueron distintas, en nueve (17\%) sólo se realizó determinación para los genes de BRCA1 y BRCA2, en 38 (71.7\%) fue un panel de 20-30 genes y en seis (11.3\%) panel de más de 30 genes. El método utilizado fue secuenciación de nueva generación con tecnología Illumina en todas las plataformas. De las que se pudieron obtener datos sobre el desempeño, la amplitud fue de -20 a
+20 nucleótidos y la profundidad de cobertura fue un poco más variable, con rangos de $20 \mathrm{x}-2000 \mathrm{x}$ y promedios reportados de $250 \mathrm{x}-1500 \mathrm{x}$.

La mutación de BRCA1 y/o BRCA2 se observó sólo en nueve/30 (30\%), todas consideradas como variantes patogénicas. La más frecuente fue la mutación en BRCA2 en cinco (55.5\%), tres (33.3\%) en BRCA1 y una (11.1\%) paciente presentó mutación de ambos genes. La mutación fundadora mexicana en BRCA1 ex9-12del se detectó en una paciente. Las variantes genéticas identificadas se describen en la Tabla 3.

Se reportaron mutaciones diferentes a BRCA en $21 / 30(70 \%)$, de ellas siete $(33.3 \%)$ fueron mutaciones patogénicas y $14(66.7 \%)$ se describieron como variantes con significado incierto. Las otras variantes genéticas identificadas se detallan en la Tabla 4. Los genes con mutaciones patogénicas fueron ATM, MUTYH y TP53 en dos mujeres cada uno y PALB2 en un solo caso.

$\mathrm{Al}$ momento del análisis nueve/48 (18.7\%) mujeres en etapa no metastásica habían mostrado recurrencia de la enfermedad. Ninguna con mutación en los genes de BRCA. Una presentaba mutación patogénica en TP53 y en dos casos se reportaron variantes de significado incierto en los genes BAP1 y 
DICER1, respectivamente. Dos $(3.8 \%)$ pacientes desarrollaron un segundo tumor primario, gástrico y sarcoma uterino indiferenciado, ninguna se detectó con mutación patógena o de significado incierto. No se encontró diferencia en el tiempo libre de enfermedad de acuerdo con la presencia o ausencia de mutaciones en BRCA o el riesgo clínico de las mutaciones.

\section{DISCUSIÓN}

En este trabajo se realizó una revisión retrospectiva de cinco años de los casos de mujeres con diagnóstico de cáncer de mama de 50 años o menos bajo seguimiento o en tratamiento activo con terapia endocrina, citotóxica o biológica con intención adyuvante, neoadyuvante o paliativa. Se identificaron 360 casos. Se encontró evidencia de solicitud de panel genético en 91 pacientes y 53 casos contaban con disponibilidad del perfil genético realizado. De los 53 casos analizados, 30 (56.6\%) presentaron alguna mutación en el panel genético. La prevalencia de mutación en los genes de BRCA en nuestra población fue de $16.9 \%$, cifra similar a la reportada en un estudio mexicano. En un análisis de Villarreal-Garza y colaboradores para determinar la prevalencia de mutaciones en BRCA1/2 de 188 mujeres mexicanas con CM y ovario sin antecedentes heredofamiliares de cáncer se informó prevalencia de $15 \%$. También en el estudio británico prospectivo $\mathrm{POSH}$, el cual incluyó 2,733 mujeres de 18 a 40 años, la prevalencia descrita fue $13 \% .14,15$

En este estudio entre las mujeres con mutación en los genes de BRCA, sólo en un caso se identificó la mutación fundadora mexicana en BRCA1 ex9-12del. Otros reportes mexicanos informan la relevancia de esta mutación específica. En una cohorte retrospectiva del Instituto Nacional de Cancerología de 188 mujeres con CM y ovario, la mutación de BRCA1 ex9-12del representó 29\% de las mutaciones encontradas. Por otro lado, en otra cohorte nacional que incluyó 190 mujeres con CM triple negativo, la prevalencia de la mutación fundadora local se observó en $41 \%$ de los casos. ${ }^{13,14}$

En el presente trabajo no se detectó una variante genética predominante, esto puede explicarse posiblemente por el tamaño de la muestra estudiada y las características de nuestra población, que si bien nuestro grupo se considera homogéneo en función de la edad, existe una amplia diversidad étnica con mujeres de ascendencia judía, europea y latina.

El alto grado histológico fue la única variable clínico-patológica que representó relevancia estadística $(\mathrm{p}=0.04)$ entre las pacientes con y sin mutación en los genes de BRCA. Esto es consistente con los resultados del estudio Prospective Outcomes in Sporadic versus Hereditary Breast Cancer (POSH), donde 82\% de las mujeres con mutación de BRCA1/2 mostraron alto grado histológico, 82 versus $56 \%$ ( $p<0.0001$ ) entre las mujeres con y sin mutación. ${ }^{15}$

En esta investigación, en el subgrupo de tumores triple negativo la prevalencia de mutaciones de BRCA1/2 fue $8.9 \%$, esta proporción parece ser menor que la reportada en otros estudios nacionales, donde se informó prevalencia de mutación en $23-27 \%$ de los casos..$^{13,14}$ Esto puede explicarse probablemente por el tamaño de la muestra y por tratarse de un estudio que incluye todos los subgrupos inmunohistoquímicos y que no se enfoca sólo en el subgrupo triple negativo.

En cuanto al tiempo a la recurrencia, en este estudio no se encontraron diferencias de acuerdo con la presencia o ausencia de mutación en BRCA. A pesar del corto tiempo de seguimiento, estos resultados concuerdan con otros reportes internacionales. En

Tabla 3: Variantes genéticas identificadas para BRCA 1/2. $(\mathrm{N}=9)$.

\begin{tabular}{|c|c|c|c|c|c|}
\hline Número & Gen & Cambio genómico & Cambio proteico & $\begin{array}{l}\text { NCBI } 1000 \\
\text { Genomas }\end{array}$ & Riesgo clínico \\
\hline 4 & BRCA 1 & c. $1960 A>T$ & p.Lys654* & rs80357355 & Patogénico \\
\hline 16 & BRCA 1 & c.815_824dupAGCCATGTGG & p.Thr276Alafs*14 & rs387906563 & Patogénico \\
\hline 38 & BRCA 1 & c. $211 A>G$ & p.Arg71Gly & rs80357382 & Patogénico \\
\hline 8 & BRCA 2 & c.658_659del & p.Val220llefs*4 & rs80359604 & Patogénico \\
\hline 24 & BRCA 2 & c. $6413 \mathrm{~T}>\mathrm{A}$ & pVal2138Asp & rs80358877 & Incierto \\
\hline 40 & BRCA 2 & c.8988_8990delATAinsTT & p.Leu2996Phefs & rs397508027 & Patogénico \\
\hline 42 & BRCA 2 & c.5146_5149de & p.Tyr1716LysFs*8 & rs276174854 & Patogénico \\
\hline 49 & BRCA 2 & c.6244del & p.Leu2082fs & rs1131691125 & Patogénico \\
\hline
\end{tabular}


Tabla 4: Otras variantes genéticas identificadas. $(\mathrm{N}=21)$.

\begin{tabular}{|c|c|c|c|c|c|}
\hline Número & Gen & Cambio genómico & Cambio proteico & $\begin{array}{c}\text { NCBI } 1000 \\
\text { Genomas }\end{array}$ & Riesgo clínico \\
\hline 45 & ATM & c. $7502 A>G$ & p.Asn2501Ser & rs531617441 & Incierto \\
\hline 48 & ATM & c. $3663 \mathrm{G}>\mathrm{A}$ & p.Trp1221 & rs864622490 & Patogénica \\
\hline \multirow{2}{*}{52} & ATM & c.2839-3_2839delinsGATACTA & & rs786202148 & Patogénica \\
\hline & APC & C. $1895 T>C$ & p.lle632Thr & rs587781360 & Incierto \\
\hline \multirow[t]{2}{*}{3} & ATM & $c .6919 C>T$ & p.Leu2307Phe & rs56009889 & Incierto \\
\hline & SMAD4 & c746_747delinsCC & p.Gln249delinsPro & rs587782209 & Incierto \\
\hline 23 & BAP1 & c. $623 G>A$ & p.Arg208Gln & rs867416499 & Incierto \\
\hline 32 & BRIP1 & c.3088_3096dup & $\begin{array}{c}\text { p.Ala1030_Ser- } \\
\text { 1032dup }\end{array}$ & rs1187782159 & Incierto \\
\hline 36 & CDKN2A & C. $146 \mathrm{~T}>\mathrm{C}$ & p.lle49Thr & rs199907548 & Incierto \\
\hline 5 & CHEK2 & c. $1567 C>T$ & p.Arg523Cys & rs149501505 & Incierto \\
\hline 30 & DICER1 & c. $1798 \mathrm{G}>\mathrm{C}$ & p.D600H & & Incierto \\
\hline 22 & FANCM & $c .5832 \mathrm{G}>\mathrm{T}$ & p.Leu1944Phe & rs201017015 & Incierto \\
\hline \multirow[t]{2}{*}{41} & $\mathrm{FH}$ & c. $1481 C>T$ & p.Ala494Val & rs752369363 & Incierto \\
\hline & TSC1 & c. $2432 \mathrm{G}>\mathrm{A}$ & p.Arg811Gln & rs761281095 & Incierto \\
\hline 11 & MLH1 & c.2219T>C & p.lle740Thr & rs1044486319 & Incierto \\
\hline 12 & MUTYH & c.1227_1228dup & p.Glu4110Glyfs 43 & rs587780078 & Patogénico \\
\hline 16 & MUTYH & c.1227_1228dupGG & p.Glu410Glyfs*43 & rs587780078 & Patogénico \\
\hline 20 & PALB2 & c.509 510del & p.Arg170llefs*14 & rs515726123 & Patogénico \\
\hline 14 & PMS2 & c. $865 \mathrm{~T}>\mathrm{A}$ & p.Phe2891le & rs771787834 & Incierto \\
\hline 47 & POLE & c. $4150 \mathrm{C}>\mathrm{T}$ & & rs756837862 & Incierto \\
\hline 6 & RAD51C & c. $492 T>G$ & p.Phe164Leu & rs573992101 & Incierto \\
\hline 17 & TP53 & $c .604 C>T$ & p.Arg202Cys & rs587780072 & Incierto \\
\hline 50 & TP53 & c. $587 \mathrm{G}>\mathrm{C}$ & p.Arg196Pro & rs483352697 & Patogénica \\
\hline \multirow[t]{2}{*}{43} & TP53 & $c .587 \mathrm{G}>\mathrm{C}$ & p.Arg196Pro & rs483352697 & Patogénica \\
\hline & KDR & c. $1416 A>T$ & p.Gln472His & rs1870377 & Incierto \\
\hline
\end{tabular}

el estudio POSH, tras un seguimiento de 8.2 (6-9.9) años, no se observaron diferencias en términos de supervivencia entre las mujeres con y sin mutación de BRCA1/2, mostrando supervivencia a dos años de 97 y $96.6 \%$, a cinco años de 83.8 y $85 \%$, y a 10 años de 73.4 y $70.1 \%$, respectivamente (HR 0.96 (IC 95\% $0.76-1.22) ; \mathrm{p}=0.76) .{ }^{15}$ Por otra parte, en un metaanálisis de 66 estudios de Van den Broek y colaboradores, cuyo objetivo fue evaluar el pronóstico de mujeres con CM y mutaciones en BRCA1/2, se concluyó que para estas mutaciones hubo una tendencia hacia una peor supervivencia global y específica por CM; sin embargo, los resultados fueron heterogéneos y la evidencia se consideró insuficiente. ${ }^{16}$

Adicionalmente, nuestro estudio reveló mutaciones diferentes a BRCA1/2 en 39.6\% de los casos estudiados (21/53). En siete/21 casos (33.3\%) se identificaron mutaciones patogénicas en los genes ATM (dos casos), MUTYH (dos casos), TP53 (dos casos) y PALB2 (un caso). En la población mexicana hay pocos estudios que reportan la frecuencia de estas mutaciones. En relación con el gen TP53 Gallardo-Alva- rado y colaboradores identificaron una prevalencia de 6.4\%. ${ }^{17}$ Para ATM, Quezada-Urban y su equipo detectaron una prevalencia de $0.3 \% .{ }^{18}$ PALB2 se ha descrito recientemente como un gen con penetrancia intermedia en la génesis del CM; Antoniou y colegas en un análisis de 152 familias con dicha mutación apuntaron un riesgo acumulado de desarrollar CM de $14 \%$ a los 50 años y de $35 \%$ a los 70 años; también se notó aumento hasta de $58 \%$ en pacientes con antecedentes heredofamiliares en primer grado de CM en menores de 50 años. ${ }^{19}$ Nuestro hallazgo puede dar pauta a futuros estudios, ya que se desconoce con exactitud cuál es su prevalencia y variantes predominantes en la población mexicana.

Las restantes 14/21 (66.7\%) pacientes presentaron mutaciones consideradas como variantes con significado incierto, ninguna de esas alteraciones se ha reportado con relevancia epidemiológica en Latinoamérica. ${ }^{20}$ Actualmente, los genes considerados con alta penetrancia de desarrollar CM familiar son: BRCA1, BRCA2, TP53, PTEN, SKT11, CDH1 y MMR. Otros genes que se han estudiado y que 
muestran una penetrancia intermedia son: CHEK2, ATM, PALB2 (FANCN), BRIP1 (FANCJ), RAD51C (FANCO), RAD51D, BARD1, MRE11, RAD50, NBS1 y FANCM. ${ }^{21}$

Este trabajo presenta algunas limitaciones metodológicas que deben ser consideradas al momento de interpretar sus hallazgos. Entre dichas limitaciones se encuentra su carácter retrospectivo; este tipo de estudios pueden tener sesgos de selección, memoria y mala clasificación. Por otro lado, para indicar la realización del estudio genético no se utilizó ningún modelo predictivo o sistema de puntuación para evaluar la probabilidad de una mutación patógena como BRCAPRO, BOADICEA o el Manchester Scoring System; entonces, la decisión para realizar el estudio genético fue en función predominantemente de la edad, la historia clínica y el criterio del médico tratante, lo que puede minimizar la frecuencia de mutaciones detectadas. Otra debilidad de este estudio es la heterogeneidad que existe entre las múltiples plataformas genéticas empleadas, que si bien todas utilizan secuenciación de nueva generación con tecnología Illumina, el desempeño (amplitud y profundidad) fue variable de acuerdo con cada plataforma y la cantidad y tipo de genes analizados fueron distintos, lo que puede representar un sesgo de ejecución. Esto podría mejorarse en un estudio prospectivo predefiniendo una sola plataforma en un grupo de pacientes preseleccionados en función de un modelo predictivo de riesgo estandarizado.

A pesar de las limitantes descritas, este trabajo posee tres importantes fortalezas. La primera es que fue el primer estudio realizado en nuestro centro sobre este tema. Segundo, se trata de una investigación basada en población del mundo real, siguiendo la práctica clínica habitual con un panorama general de la prevalencia de mutaciones en el amplio espectro del CM. Tercera, el estudio fue diseñado y calculado para obtener un nivel de confianza de sus resultados de $80 \%$. Adicionalmente, este trabajo nos permitió identificar otras mutaciones distintas a BRCA, lo cual aporta nueva información no disponible en población mexicana y ayuda a generar hipótesis para el desarrollo de nuevas investigaciones en esta área poco explorada.

Si bien este estudio nos ha permitido conocer la prevalencia de mutación en los genes de BRCA1/2 y distinguir que no hay diferencias en cuanto a las características clínico-patológicas, frecuencia de recurrencia y tiempo a la recurrencia, el estudio de mutaciones de BRCA y otros genes, debe continuarse con carácter prospectivo en grupos de pacientes pre- seleccionadas por modelos de riesgo a fin de conocer la verdadera prevalencia de mutaciones y su impacto en el pronóstico. Además, dicha información es de relevancia para la implementación de estrategias de prevención de segundas neoplasias en las pacientes y consejo genético para toda la familia.

Por último, otro aspecto importante que enmarca la necesidad de conocer el estado mutacional de los genes de BRCA se enfoca en el grupo de pacientes con enfermedad recurrente a distancia, para esos casos se ha desarrollado un grupo de medicamentos que tienen como blanco terapéutico el bloqueo de la vía donde intervienen los genes de BRCA1/2. Los inhibidores de PARP han demostrado un importante beneficio en mujeres con CM metastásico y mutación BRCA1/2 (OlympiAD, EMBRACA). ${ }^{22,23}$ Actualmente, algunos ensayos clínicos están en curso para probar la eficacia de estos fármacos en etapas no metastásicas (OlympiA, SUBITO). ${ }^{24}$

\section{CONCLUSIONES}

En este estudio, la prevalencia de mutaciones en BRCA1/2 en mujeres menores de 50 años con cáncer mamario es de $16.9 \%$, además, $13.2 \%$ mostró mutación en otros genes de alta e intermedia penetrancia (ATM, MUTYH, TP53 y PALB2). En el presente estudio no se observaron diferencias en las características clínico-patológicas entre los grupos con y sin mutación de BRCA1/2, excepto en el alto grado de diferenciación, el cual fue más frecuente en el grupo mutado. Por otro lado, en este trabajo no detectaron diferencias en la frecuencia de recurrencia ni tiempo a la recurrencia entre los grupos con y sin mutación. Se requiere de otros estudios prospectivos, con mayor número de muestra y población homogénea para confirmar los hallazgos de esta investigación.

\section{REFERENCIAS}

1. Bray F, Ferlay J, Soerjomataram I, Siegel RL, Torre LA, Jemal A. Global cancer statistics 2018: GLOBOCAN estimates of incidence and mortality worldwide for 36 cancers in 185 countries. CA Cancer J Clin. 2018; 68 (6): 394-424.

2. Villarreal-Garza C, Aguila C, Magallanes-Hoyos MC et al. Breast cancer in young women in Latin America: an unmet, growing burden. Oncologist. 2013; 18 (12): 1298-1306.

3. Rodríguez-Cuevas S, Macías CG, Franceschi D, Labastida S. Breast carcinoma presents a decade earlier in Mexican women than in women in the United States or European countries. Cancer. 2001; 91: 863-868.

4. Balic M, Thomssen C, Würstlein R, Gnant M, Harbeck N. St. Gallen/Vienna 2019: a brief summary of the consensus discussion on the optimal primary breast cancer treatment. Breast Care (Basel). 2019; 14: 103-110. 
5. Fu J, Wu L, Fu W et al. How young is too young in breast cancer?-young breast cancer is not a unique biological subtype. Clin Breast Cancer. 2018; 18 (1): e25-e39.

6. Pollán M. Epidemiology of breast cancer in young women. Breast Cancer Res Treat. 2010; 123 (Suppl 1): 3-6.

7. Gabriel CA, Domchek SM. Breast cancer in young women. Breast Cancer Res. 2010; 123 (Suppl 1): 212.

8. Keegan TH, DeRouen MC, Press DJ, Kurian AW, Clarke CA. Occurrence of breast cancer subtypes in adolescent and young adult women. Breast Cancer Res. 2012; 14 (2): R55.

9. Kroman N, Jensen MB, Wohlfahrt J, Mouridsen HT, Andersen PK, Melbye M. Factors influencing the effect of age on prognosis in breast cancer: population based study. BMJ. 2000; 320 (7233): 474-478.

10. Stoppa-Lyonnet D. The biological effects and clinical implications of BRCA mutations: where do we go from here? Eur J Hum Genet. 2016; 24 (Suppl 1): S3-S9.

11. Antoniou AC, Pharoah PD, McMullan G et al. A comprehensive model for familial breast cancer incorporating BRCA1, BRCA2 and other genes. Br J Cancer. 2002; 86 (1): 76-83.

12. Sapkota Y. Germline DNA variations in breast cancer predisposition and prognosis: a systematic review of the literature. Cytogenet Genome Res. 2014; 144 (2): 77-91.

13. Villarreal-Garza C, Weitzel JN, Llacuachaqui M et al. The prevalence of BRCA1 and BRCA2 mutations among young Mexican women with triple-negative breast cancer. Breast Cancer Res Treat. 2015; 150 (2): 389-394.

14. Villarreal-Garza C, Álvarez-Gómez RM, Pérez-Plasencia C et al. Significant clinical impact of recurrent BRCA1 and BRCA2 mutations in Mexico. Cancer. 2015; 121 (3): 372-378.

15. Copson ER, Maishman TC, Tapper WJ et al. Germline BRCA mutation and outcome in young-onset breast cancer (POSH): a prospective cohort study. Lancet Oncol. 2018; 19 (2): 169-180.
16. Van den Broek AJ, Schmidt MK, van 't Veer LJ, Tollenaar RA, Van Leeuwen FE. Worse breast cancer prognosis of BRCA1/BRCA2 mutation carriers: what's the evidence? A systematic review with meta-analysis. PLoS One. 2015; 10: e0120189.

17. Gallardo-Alvarado LN, Tusié-Luna MT, Tussié-Luna MI et al. Prevalence of germline mutations in the TP53 gene in patients with early-onset breast cancer in the Mexican population. BMC Cancer. 2019; 19 (1): 118.

18. Quezada UR, Díaz VCE, Gitler R et al. Comprehensive analysis of germline variants in mexican patients with hereditary breast and ovarian cancer susceptibility. Cancers (Basel). 2018; 10 (361): 1-19.

19. Antoniou AC, Foulkes WD, Tischkowitz M. Breast-cancer risk in families with mutations in PALB2. N Engl J Med. 2014; 371 (17): 1651-1652.

20. Ashton-Prolla P, Vargas FR. Prevalence and impact of founder mutations in hereditary breast cancer in Latin America. Genet Mol Biol. 2014; 37 (1 Suppl): 234-240.

21. Economopoulou P, Dimitriadis G, Psyrri A. Beyond BRCA: new hereditary breast cancer susceptibility genes. Cancer Treat Rev. 2015; 41: 1-8.

22. Robson ME, Tung N, Conte $\mathrm{P}$ et al. OlympiAD final overall survival and tolerability results: Olaparib versus chemotherapy treatment of physician's choice in patients with a germline BRCA mutation and HER2-negative metastatic breast cancer. Ann Oncol. 2019; 30 (4): 558-566.

23. Litton JK, Rugo HS, Ettl J et al. Talazoparib in patients with advanced breast cancer and a germline BRCA mutation. N Engl J Med. 2018; 379: 753-763.

24. Goncalves A, Bertucci A, Bertucci F. PARP inhibitors in the treatment of early breast cancer: the step Beyond? Cancers (Basel). 2020; 12: 1378 . 